Materials and Methods We tested, using a 5 methyl cytosine (5MeCyt) ELISA, global DNA methylation in long-term cultured salivary gland epithelial cells (SGEC), peripheral T cells and B cells from eight SS patients. DNA methylation/demethylation partners were assessed by real time quantitative PCR (DNA methyl transferase (DNMT)1, DNMT3a/b, PCNA, UHRF1, MBD2, MBD4, and Gadd45-alpha). Immunofluorescence was conducted on labial salivary gland biopsy. Co-culture experiments were performed associating the human salivary gland cell line (HSG) and B cells.

Results Global DNA methylation was reduced in SGEC from SS patients (5MeCyt: $36.3 \pm 3.2 \%$ in SS versus $43.1 \pm 3.3 \%$ in controls, $P=0.01$ ), while no difference was observed in $\mathrm{T}$ and $\mathrm{B}$ cells. SGEC demethylation in SS patients was associated with a 7-fold decrease of DNMT1 and a 1.8-fold increase of Gadd45-alpha expression. The other DNA methylation/demethylation partners tested were not differently expressed when compared to controls. Interestingly, SGEC demethylation may be attributed to the B cell infiltrate as DNA methylation increased in salivary gland biopsy after rituximab (anti-CD20 antibody) treatment. Such hypothesis was confirmed using co-culture experiments (HSG cells and B cells) revealing an alteration of the PKC-delta/ERK/DNMT1 pathway. Finally, DNA methylation was associated with the overexpression of several SGEC genes such as ICAM-1 and human endogenous retrovirus (HERV).

Conclusions SGEC dysfunction in SS may be linked to epigenetic modifications and this tissue specific defect may be ascribed in part to infiltrating B cells. This observation opens new therapeutic perspectives in SS.

\section{A7.9 DOES TELOMERE SHORTENING IN WOMEN WITH RHEUMATOID ARTHRITIS PREDICT X CHROMOSOME INACTIVATION BIAS?}

doi:10.1136/annrheumdis-2013-203221.9

${ }^{1}$ Sami Barna Kanaan, ${ }^{2}$ Onur Emre Onat, 1,3Nathalie Balandraud, 'Doua F Azzouz, ${ }^{1,3} \mathrm{Jean}$ Roudier, ${ }^{T}$ Tayfun Ozcelik, 'Nathalie C Lambert. 'INSERM UMR 1097, Marseille, France; ${ }^{2}$ Department of Molecular Biology and Genetics, Bilkent University, Ankara, Turkey; ${ }^{3}$ Rheumatology Department, Hôpital Sainte Marguerite, Marseille, France

Background Rheumatoid Arthritis (RA), like most auto-immune diseases, is a female predominant disease. As a possible explanation for gender bias, we have previously shown that women with RA have non-random X chromosome inactivation (XCI) that could trigger autoimmunity (article in preparation). Intriguingly, this bias in XCI correlates with presence of the shared epitope (SE) and with disease duration.

Also associated with presence of the SE, premature immunosenescence, characterised by shorter telomere length, has been described in peripheral blood cells from patients with RA [1]. Moreover, telomeric non coding RNAs have been reported to be enriched near the inactive $\mathrm{X}$ chromosome in mammals [2] indicating a potential link between telomere length and XCI.

Objectives In this context, we propose to test whether women with RA have shortened telomere length and whether that could influence the epigenetic mechanism of XCI.

Methods A total of 73 women with RA and 48 healthy women with no history of autoimmune diseases, who had previously been tested for XCI and HLA-genotyped, were evaluated for telomere length. The relative telomere length was estimated by real-time PCR as originally described by Cawthon [3] with the $2^{-\Delta \Delta \mathrm{Ct}}$ method. Results Preliminary results show that women with RA have smaller telomere length than healthy women, although the difference is modest $(p=0.07)$ and has to be adjusted for age on a larger cohort. Contrary to expectations, shorter telomere length is not correlated with skewed XCI status, disease duration or the presence of shared epitope in our small cohort.
Conclusions This preliminary study seems to confirm that women with RA have shorter telomeres than healthy women. Further telomere length measurements have to be done on a larger group of patients with RA and healthy controls, as well as HLAgenotyping them and evaluating their XCI status. This will be a step forward in understanding the relationship between immune senescence, female predisposition and genetic risk (SE) in RA.

References

1. SO Schonland et al, Proc Natl Acad Sci U S A 100, 13471 (Nov 11, 2003).

2. S Schoeftner, M A Blasco, Nat Cell Biol 10, 228 (Feb, 2008).

3. RM Cawthon, Nucleic Acids Res 30, e47 (May 15, 2002).

\section{A7.10 GENETIC VARIANTS IN THE IL-4 AND IL-4 RECEPTOR GENES IN ASSOCIATION WITH THE SEVERITY OF JOINT DAMAGE IN RHEUMATOID ARTHRITIS: A STUDY IN SEVEN COHORTS}

doi:10.1136/annrheumdis-2013-203221.10

${ }^{1} \mathrm{~A}$ Krabben, ${ }^{2} \mathrm{AG}$ Wilson, ${ }^{1} \mathrm{DPC}$ de Rooy, ${ }^{1,3} \mathrm{~A}$ Zhernakova, ${ }^{4} \mathrm{E}$ Brouwer, ${ }^{5} \mathrm{E}$ Lindqvist, ${ }^{5} \mathrm{~T}$ Saxne, ${ }^{1} \mathrm{G}$ Stoeken, ${ }^{1} \mathrm{JAB}$ van Nies, 'R Knevel, 'TWJ Huizinga, ${ }^{6} \mathrm{~B}$ Koeleman, 'R Toes, ${ }^{7}$ PK Gregersen, 'AHM van der Helm-van Mil. 'Department of Rheumatology, Leiden University Medical Center, The Netherlands; ${ }^{2}$ Department of Musculoskeletal Sciences, University of Sheffield, Sheffield, UK; ${ }^{3}$ Genetics Department, University of Groningen, University Medical Center, Groningen, The Netherlands; ${ }^{4}$ Department of Rheumatology and Clinical Immunology, University of Groningen, University Medical Center, Groningen, The Netherlands; 'Department of Rheumatology, Lund University, Skåne University Hospital, Lund, Sweden; ${ }^{6}$ Department of Medical Genetics, Complex Genetics Section, Utrecht, The Netherlands; 'Feinstein Institute for Medical Research and North Shore-Long Island Jewish Health System, Manhasset, New York, USA

Objective The progression of joint destruction in rheumatoid arthritis (RA) is determined by genetic factors. Changes in $I L-4$ and $I L-4 R$ genes have been associated with RA severity but not replicated. We studied the association between $I L-4$ and $I L-4 R$ tagging SNPs and progression rate of joint damage in RA in a multi-cohort candidate gene study.

Methods $I L-4$ and $I L-4 R$ tagging SNPs (8 and 39, respectively) were genotyped in 600 RA-patients of whom 2,846 sets of hands and feet X-rays were collected during 7 years follow-up. Subsequently, significantly associated SNPs were genotyped and studied in relation to 3,415 X-rays of 1,953 RA-patients; these included data-sets from Groningen (NL), Lund (SE), Sheffield (UK), NARAC (USA), Wichita (USA) and NDB (USA). The relative increase in progression rate per year in the presence of a genotype was determined in each cohort. An inverse variance weighting meta-analysis was done on the six datasets that together formed the replication-phase.

Results In the discovery-phase none of the $I L-4$ SNPs and seven of the $I L-4 R$ SNPs were significantly associated with joint damage progression rate. In the replication-phase, two SNPs in $I L-4 R$ gene were significantly associated with joint damage progression rate (Rs1805011, $p=0.02$ and Rs1119132, $p=0.001$ ).

Conclusions Genetic variants in $I L-4 R$ were identified and independently replicated to associate with progression rate of joint damage in RA.

\section{A7.11 GENETIC VARIATION IN PROMOTER SEQUENCE OF B-CELL-ACTIVATING FACTOR OF THE TNF FAMILY (BAFF) IN PATIENTS WITH IDIOPATHIC INFLAMMATORY MYOPATHIES (IIM)}

doi:10.1136/annrheumdis-2013-203221.11

'M Faustova, 'L Plestilova, ${ }^{1} \mathrm{H}$ Hulejova, ${ }^{2} \mathrm{O}$ Pecha, ${ }^{3} \mathrm{Z}$ Betteridge, ${ }^{1} \mathrm{H}$ Mann, ${ }^{1} \mathrm{P}$ Putova, 'J Vencovsky, 'P Novota, '0 Krystufkova. 'Institute of Rheumatology and Department of Rheumatology, 1 st Faculty of Medicine, Charles University, Prague; 'Institute of Biophysics and Informatics, 1st Faculty of Medicine, Charles University Prague; ${ }^{3}$ Rheumatology Department, Royal National Hospital for Rheumatic Diseases, Bath, UK 
Background and Objectives B-cell activating factor of the TNF family (BAFF) is important for B cell maturation and plays a role in (auto)antibodies production. Elevated serum levels in relation to autoantibodies were documented in patients with IIM. Promoter region of BAFF gene contains several known sites with single nucleotide polymorphism (SNPs). An association between Rs9514828 (-871 C/T) SNP and susceptibility to idiopathic trombocytopaenic purpura was shown and possible relations to systemic lupus erythematosus, rheumatoid arthritis or Sjögren's syndrome were suggested but in IIM have not been studied yet. Here, we analysed relation of four BAFF SNPs located in the BAFF gene promoter with the development of IIM.

Materials and Methods 146 patients with polymyositis (PM), 150 with dermatomyositis (DM), 11 patients with juvenile DM and 4 patients with inclusion body myositis and 103 healthy individuals were included. Four SNPs located upstream in the BAFF gene (Rs9514827 (-2841 T/C); Rs3759467 (-2704 T/C); Rs1041569 (-2701 T/A); Rs9514828 (-871 C/T)) were analysed by direct DNA sequencing. Serum levels of BAFF (s-BAFF) were evaluated using ELISA. Autoantibodies were detected with immunoprecipitation. The chi square test for analysis of alleles and genotypes associations and SNPStats software for haplotype frequency studies were used. Results Significantly higher frequency of $-2701 \mathrm{~T}$ allele was present in patients $(18 \%)$ compared to healthy controls $(12 \%)(\mathrm{P}=0.029$; OR $1.684(\mathrm{CI} 95 \%=1.050-2.699))$. Additionally, increased $-2841 \mathrm{~T}$ allele $(\mathrm{P}=0.086),-2841 \mathrm{TT}$, CT genotype $(\mathrm{P}=0.066)$ and $-2701 \mathrm{TT}$, AT genotype $(\mathrm{P}=0.079)$ frequencies were observed in patients. SNPs were in strong linkage disequilibrium and formed four common haplotypes (TTAC, CTAT, TCAC, TTTT), with significantly different frequency $(>9 \%)$ distributions between patients and controls (global P-value <0.038). Higher frequency of TTTT haplotype was present in patients $(16.2 \%)$ compared to controls $(9.3 \%$; OR 1.99 (95\% CI 1.15-3.47; $\mathrm{P}<0.015)$ ) relative to the most frequent haplotype TTAC. Significantly higher s-BAFF levels were detected in patients compared to healthy controls $(\mathrm{P}<0.0001)$ and in patients with anti-Jo-1 or anti-PMScl autoantibodies compared to patients without autoantibodies ( $\mathrm{P}=0.028, \mathrm{P}<0.037$ respectively). Higher s-BAFF levels with $-2704 \mathrm{~T}$ allele within anti-Jo-1+ patients $(\mathrm{P}<0.043)$ were found.

Conclusions We describe significant association of SNP (Rs1041569) with myositis and a relation of SNP (Rs3759467) to presence of anti-Jo-1 autoantibodies and s-BAFF levels. These results should be confirmed in an independent cohort of patients and possible relations of s-BAFF levels with disease activity and treatment should be considered.

Acknowledgement IGA -Ministry of Health of the Czech Republic NT/12438-4.

\section{A7.12 IDENTIFICATION OF THE SEMAPHORIN RECEPTOR PLEXIN-A2 AS A CANDIDATE GENE FOR SUSCEPTIBILITY TO ANKYLOSING SPONDYLITIS}

doi:10.1136/annrheumdis-2013-203221.12

A Chatzikyriakidou, PV Voulgari, AA Drosos. Rheumatology Clinic, Department of Internal Medicine, Medical School, University of loannina, Greece

Background and Objectives Semaphorins are a large family of secreted and membrane bound proteins linked to their receptors in a variety of cells and implicated with the development and function of many systems (nevrous, immune, vascular) and organs (bone, heart, lung, kidney). Plexins and neuropilins are the primary semaphorin receptors. The present study addresses Plexin-A2 (PLXNA2) Rs6656560 variant in rheumatoid arthritis (RA), ankylosing spondylitis (AS), and psoriatic arthritis (PsA) susceptibility.

Materials and Methods One hundred thirty-six RA, 49 AS, 29 PsA patients and 147 controls were enrolled in the study. Polymerase chain reaction coupled with restriction fragment length polymorphism analysis was used in Rs6656560 genotyping.

Results Statistical significant difference was observed in Rs6656560 genotypes' distribution between AS patients and controls $(P=0.041)$, but not between RA or PsA patients and controls $(\mathrm{P}=0.183$, $\mathrm{P}=0.729$; respectively). Similarly, significant difference was observed in Rs6656560 alleles' distribution between AS patients and controls $(\mathrm{P}=0.013)$. Since AS and PsA both belong to spondyloarthropathies (SpAs), no statistical difference was observed in genotypes' distribution between these two groups $(\mathrm{P}=0.178)$, while the statistical difference was significant between AS and RA patients $(\mathrm{P}=0.035)$.

Conclusions The positive association of PLXNA2 polymorphism with AS susceptibility seems to indicate its effect in cellular semaphorin signalling related to bone development and remodelling, both of which are implicated in AS features. Additional studies are needed to ensure the revealed genetic association with AS predisposition and the effect of this variant in semaphorin/plexin complex function in specific cell types.

\section{A7.13 IRAK1 RS3027898 POLYMORPHISM: A VARIANT IMPLICATED IN THE PATHOGENESIS OF MORE THAN ONE INFLAMMATORY DISEASE}

doi:10.1136/annrheumdis-2013-203221.13

A Chatzikyriakidou, PV Voulgari, AA Drosos. Rheumatology Clinic, Department of Internal Medicine, Medical School, University of loannina, Greece

Background and Objectives IRAK1 plays significant role in TLR dependent activation of the transcription factor NF-kB, which subsequently increases the expression of many genes such as TNF- $\alpha$ and IL-8 related to immunological reactions. Polymorphism Rs3027898 located in the 3'-untranslated region of IRAK1 gene was studied for its association with rheumatoid arthritis (RA), psoriatic arthritis (PsA) and ankylosing spondylitis (AS) predisposition.

Materials and Methods The polymerase chain reaction-single strand conformation polymorphism analysis coupled with sequencing was used as the screening method for variant genotyping in 136 RA, 29 PsA, and 49 AS patients and 147 controls.

Results IRAK1 polymorphism Rs3027898 was revealed to be associated with all the studied inflammatory conditions. Specifically, strong statistically significant difference was observed in polymorphism distribution between RA patients and controls $(p=0.044)$, PsA patients and controls $(p<0.001)$, and AS patients and controls $(p<0.001)$. Grouping genotypes AA and AC versus $\mathrm{CC}$ the statistical difference was also significant in all groups: RA versus controls $(p=0.017)$, PsA versus controls $(p<0.001)$, and AS versus controls $(p=0.010)$.

Conclusions Here, we report IRAK1 gene polymorphism association with RA, PsA, and AS. This variant, previously, was also related to RA in patients with Chinese origin, with atherothrombotic cerebral infarction in Japanese patients, while we also revealed its association with ischemic stroke. In addition, another IRAK1 gene polymorphism (Rs1059703) was also related to atherothrombotic cerebral infraction, high CRP, chronic kidney disease, the induction of vaccine-induced immunity and sepsis outcomes but this variant did not differ significantly between our studied groups and controls (data not given). Taking into account that IRAK1 gene affects the activation of transcription factor NF-kB, which is implicated in many immune related genes' expression, we could understand IRAK1 extensive association with many inflammatory conditions beyond patients' origin.

\section{A7.14 IRF5 POLYMORPHISMS IN SYSTEMIC SCLEROSIS}

doi:10.1136/annrheumdis-2013-203221.14

${ }^{1} \mathrm{C}$ Stock, ${ }^{1} \mathrm{H}$ Sato, ${ }^{2} \mathrm{C}$ Fonseca, ${ }^{1} \mathrm{AU}$ Wells, ${ }^{2} \mathrm{CP}$ Denton, ${ }^{2} \mathrm{DJ}$ Abraham, ${ }^{1} \mathrm{G}$ Lindahl, 'EA Renzoni. 'Interstitial Lung Disease Unit, Royal Brompton Hospital, London, UK; ${ }^{2}$ Center for Rheumatology, Royal Free Hospital, London, UK 